\title{
UNIQUE CONTINUATION FOR A CLASS OF HIGHER ORDER ELLIPTIC OPERATORS
}

\author{
Laura De CARLi
}

In this paper we prove a unique continuation theorem for a class of elliptic operators of order $m \geq 2$ with highly singular potentials using the method of Carleman estimates.

\section{Introduction.}

In this paper we establish a unique continuation theorem for solutions of a class of differential inequalities of the form:

$$
|P(D) U(x)| \leq|V(x) U(x)|,
$$

where $P$ is an elliptic operator with constant coefficients and $V$ is a singular potential.

We will prove that if $n>m, P$ is an elliptic operator of order $m \geq 2$ whose principal part satisfies conditions that will be specified later, $V \in L^{\frac{n}{m}}\left(\mathbb{R}^{n}\right)$, and if $U \in H^{m, p}\left(\mathbb{R}^{n}\right), p=\frac{2 n}{m+n}$, satisfies (0.1), then $U$ is identically zero if its support is contained in a half space whose normal direction satisfies a hypothesis involving the symbol of $P$. By $H^{m, p}\left(\mathbb{R}^{n}\right)$ we mean the space of functions with $m$ derivatives in $L^{p}\left(\mathbb{R}^{n}\right)$.

It is well known that the above unique continuation property, (u.c.p. henceforth in this paper), for the solutions of the differential Inequality (0.1), follows from the proof of a weighted inequality of the form

$$
\left\|e^{\tau \phi(x)} u\right\|_{p^{\prime}} \leq C\left\|e^{\tau \phi(x)} P(D) u\right\|_{p},
$$

valid for all $u \in H^{m, p}\left(\mathbb{R}^{n}\right)$, a suitable weight $\phi$ depending on the half space, and values of the real parameter $\tau$ which are allowed to tend to $+\infty$. An estimate of the form of (0.2) is called a Carleman-type inequality.

There is a lot of literature concerning Carleman-type inequalities and unique continuation properties for solutions of partial differential equations. See $[\mathbf{H} \mathbf{1}],[\mathbf{J}],[\mathbf{S} \mathbf{1}]$, just to cite a few. When $m=2$, the u.c.p. for the solutions of (0.1) has been proved in $[\mathrm{KRS}]$. Moreover, the assumption that $u$ vanishes on a open set of $\mathbb{R}^{n}$ can be replaced by the much weaker assumption that $u$ vanishes of infinite order at some point in $\mathbb{R}^{n}$. See $[\mathbf{J K}]$. 
In their paper the authors proved also that the hypothesis $V \in L_{\text {loc }}^{\frac{n}{2}}\left(\mathbb{R}^{n}\right)$ is optimal, in the sense that $\frac{n}{2}$ cannot be replaced by any smaller exponent.

Let $u \in H^{m, p}\left(\mathbb{R}^{n}\right)$ be a solution of (0.1). Suppose that $u$ is supported in the half space $\{x:\langle x \nu\rangle \geq 0\}, \nu$ being a unit vector of $\mathbb{R}^{n}$. Our paper will be devoted to the proof of $(0.2)$, with $\phi(x)=-\langle x \nu\rangle$. A standard argument, that will be recalled in the next section, will prove that the u.c.p. holds in $H^{m, p}\left(\mathbb{R}^{n}\right)$ for the solutions of $(0.1)$.

To prove (0.2), and to explain the assumptions that the principal part of $P$ must satisfy, we shall make some standard reductions. For simplicity we assume that $P$ is homogeneous, since the other cases follow from easy adaptions of this argument. If we define the conjugate operator of $P$ as

$$
P_{\tau}(D) u=e^{\tau\langle x \nu\rangle} P(D) e^{-\tau\langle x \nu\rangle}=P(D+i \tau \nu),
$$

then (0.2) will be a consequence of the following uniform inequality

$$
\|u\|_{p^{\prime}} \leq C\|P(D+i \tau \nu) u\|_{p} .
$$

Since the inverse Fourier transform of $(P(\zeta+i \tau \nu))^{-1}$ is a fundamental solution for the operator $P(D+i \tau \nu)$, the inequality (0.3) will be a consequence of

$$
\left\|\int_{\mathbb{R}^{n}} \frac{e^{i\langle\zeta, x\rangle}}{P(\zeta+i \tau \nu)} \hat{f}(\zeta) d \zeta\right\|_{p^{\prime}} \leq C\|f\|_{p}, \quad f \in \mathcal{S}\left(\mathbb{R}^{n}\right) .
$$

We now make the following important assumption:

(1) $P(D)$ has simple complex characteristics in the direction $\nu$, in the sense that the polynomial $\tau \rightarrow P(\zeta+i \tau \nu), \tau \in \mathbb{C}$, has only simple zeroes for each fixed $\zeta \in \mathbb{R}^{n} /\{0\}$.

Without loss of generality $\nu=(1,0, \ldots, 0)$. After rescaling, we can assume $\tau=1$. Let $P(\zeta)$ be the symbol of $P(D)$. After perhaps a change of coordinates,

$$
P(\zeta)=\zeta_{1}^{m}+\sum_{j=0}^{m-1} \zeta_{1}^{j} Q_{j}\left(\zeta^{\prime}\right)
$$

where the $Q_{j}$ 's are homogeneous polynomials of degree $m-j$, and where we have set $\zeta=\left(\zeta_{1}, \zeta^{\prime}\right)$. The roots of $\zeta_{1} \rightarrow P(\zeta), \lambda_{1}\left(\zeta^{\prime}\right)+i \mu_{1}\left(\zeta^{\prime}\right), \ldots$, $\lambda_{m}\left(\zeta^{\prime}\right)+i \mu_{m}\left(\zeta^{\prime}\right)$, are smooth and homogeneous of degree 1 in $\mathbb{R}^{n-1} /\{0\}$. Since $P(D)$ is elliptic with real coefficients, the $\mu_{j}$ 's vanish only at the origin, and $\lambda_{j}\left(\zeta^{\prime}\right)+i \mu_{j}\left(\zeta^{\prime}\right)$ and $\lambda_{j}\left(\zeta^{\prime}\right)-i \mu_{j}\left(\zeta^{\prime}\right)$ are both roots of $\zeta_{n} \rightarrow P\left(\zeta^{\prime}, \zeta_{n}\right)$. Then,

$$
P(\zeta)=\Pi_{j=1}^{\frac{m}{2}}\left(\zeta_{1}-\lambda_{j}\left(\zeta^{\prime}\right) \pm i \mu_{j}\left(\zeta^{\prime}\right)\right)
$$


where $\mu_{j}\left(\zeta^{\prime}\right)>0, \zeta^{\prime} \in \mathbb{R}^{n-1} /\{0\}$, and hence

$$
P(\zeta+i \nu)=\Pi_{j=1}^{\frac{m}{2}}\left(\zeta_{1}-\lambda_{j}\left(\zeta^{\prime}\right)\right)+i\left(1 \pm i \mu_{j}\left(\zeta^{\prime}\right)\right) .
$$

The factors of the form $\zeta_{1}-\lambda_{j}\left(\zeta^{\prime}\right)+i\left(1+\mu_{j}\left(\zeta^{\prime}\right)\right)$ are the "good" factors which never vanish, but the "bad" factors $\zeta_{1}-\lambda_{j}\left(\zeta^{\prime}\right)+i\left(1-\mu_{j}\left(\zeta^{\prime}\right)\right)$ vanish on the (compact) manifolds

$$
S_{j}=\left\{\zeta: \zeta_{1}=\lambda_{j}\left(\zeta^{\prime}\right), 1=\mu_{j}\left(\zeta^{\prime}\right)\right\}, \quad j=1, \ldots, \frac{m}{2} .
$$

By (1), $S_{i} \cap S_{j}=\emptyset$ when $i \neq j$. Let $\chi_{j}(\zeta) \in C_{0}^{\infty}\left(\mathbb{R}^{n}\right)$ be a cutoff function which is $\equiv 1$ in a neighborhood of $S_{j}$ and is $\equiv 0$ on a neighborhood of $S_{k}$, $k \neq j$. Let $\chi_{0}(\zeta)=1-\left(\chi_{1}(\zeta)+\cdots+\chi_{\frac{m}{2}}(\zeta)\right)$. Since $P(\zeta+i \nu)$ does not vanish on the support of $\chi_{0}, \frac{\chi_{0}(\zeta)}{P(\zeta+i \nu)}$ is the symbol of a pseudifferential operator of order $-m$, which can thus be extended to a bounded operator from $L^{p}$ to $H^{m, p}\left(\mathbb{R}^{n}\right)$ (see e.g. $[\mathbf{H}]$ ). By the Sobolev embedding theorem,

$$
\left\|\int_{\mathbb{R}^{n}} \frac{e^{i\langle x, \zeta\rangle} \hat{f}(\zeta)}{P(\zeta+i \nu)} \chi_{0}(\zeta) d \zeta\right\|_{p^{\prime}} \leq C\|f\|_{p} .
$$

We also observe that $\zeta \rightarrow \frac{\zeta_{1}-\lambda_{j}\left(\zeta^{\prime}\right)+i\left(1-\mu_{j}\left(\zeta^{\prime}\right)\right)}{P(\zeta+i \nu)} \chi_{j}(\zeta), j=1, \ldots, \frac{m}{2}$, is a $L^{p}$ Fourier multiplier for every $p>1$ (see e.g. $[\mathbf{H}]$ ). We have then reduced matters to proving the following inequalities

$$
\left\|\int_{\mathbb{R}^{n}} \frac{\hat{f}(\zeta) e^{i\langle x, \zeta\rangle} \chi_{j}(\zeta) d \zeta}{\zeta_{1}-\lambda_{j}(\zeta)+i\left(1-\mu_{j}\left(\zeta^{\prime}\right)\right)}\right\|_{p^{\prime}} \leq C\|f\|_{p}, \quad j=1, \cdots \frac{m}{2}, \quad f \in \mathcal{S}\left(\mathbb{R}^{n}\right) .
$$

Our main results can now be stated as follows.

Theorem 1. Let $P(D)$ be an elliptic operator of order $m<n$ with constant coefficients. Let $\lambda_{j}$ and $\mu_{j}$ be defined as above. Suppose that the assumption (1) is satisfied. Suppose also that

$$
\begin{aligned}
& \text { the cospheres }\left\{\zeta^{\prime}: \mu_{j}\left(\zeta^{\prime}\right)=1\right\} \text { have everywhere } \\
& \text { nonvanishing Gaussian curvature. }
\end{aligned}
$$

Then, for every $f \in C_{0}^{\infty}\left(\mathbb{R}^{n}\right)$ and for $p \leq \frac{2 n}{n+2}$, (0.5) holds.

Theorem 2. Let $P(D)$ be as in Theorem 1, and let $U \in H^{m, p}\left(\mathbb{R}^{n}\right), p=$ $\frac{2 n}{n+m}$, be a solution of the differential inequality (0.1), with $V \in L^{\frac{n}{m}}\left(\mathbb{R}^{n}\right)$. Suppose that the support of $U$ is contained on one side of a hyperplane, 
and that $P(D)$ satisfies (1) in the direction $\nu$ of the exterior normal to the hyperplane containing the support of $U$. Suppose also that (2) holds. Then $U \equiv 0$.

The Assumption (1) is crucial. The counterexamples of Plis $[\mathbf{P}]$ show in fact that the u.c.p. across the hyperplane $\{x:\langle x \nu\rangle=0\}$ can fail for solutions of elliptic operators that do not have simple complex characteristics in the direction $\nu$.

The Assumption (2) might be not necessary. An early result due to Hörmander [H2] shows in fact that the u.c.p. holds in $H^{m}\left(\mathbb{R}^{n}\right)$ for the solutions of (0.1) when $V \in L^{\infty}\left(\mathbb{R}^{n}\right)$ and (1) is satisfied. The same is probably also true in the $L^{p}$ setting. However, our theorem represents a natural generalization of the case $m=2$. When $P(D)$ is the Laplacean, we can see that the "bad factor" of the symbol of the conjugate operator $P(D+i(1,0, \ldots 0))$ is $\zeta_{1}+i\left(1-\left|\zeta^{\prime}\right|\right)$, and the Condition (2) is then satisfied.

In what follows we shall use the convention that $\chi$ denotes a smooth cutoff function which is not necessarily the same at each occurrence. Also, we will denote by $C$ a constant which may change from line to line.

This paper is a part of my doctoral thesis at UCLA. I would like to thank my teacher and adviser, Prof. C.D. Sogge for his invaluable counsel, and the referee for his constructive comments.

\section{Section 1.}

In this section we will prove our main results. First of all, we proceed as in $[\mathbf{K R S}]$ to show that Theorem 1 implies Theorem 2. Let $U \in H^{m, p}\left(\mathbb{R}^{n}\right)$ be a solution of (0.1). For simplicity of notation, we shall assume that $U$ is supported in the half space $\left\{x=\left(x_{1}, x^{\prime}\right): x_{1}>0\right\}$, since the argument for the other cases is similar. We have shown in the Introduction that Theorem 1 implies the following special case of (0.2):

$$
\left\|e^{-\tau x_{1}} u\right\|_{L^{p^{\prime}}\left(\mathbb{R}^{n}\right)} \leq C\left\|e^{-\tau x_{1}} P(D) u\right\|_{L^{p}\left(\mathbb{R}^{n}\right)}, \quad u \in C_{0}^{\infty}\left(\mathbb{R}^{n}\right) .
$$

Since $\tau x_{1} \geq 0$ on the support of $U$, and since $C_{0}^{\infty}\left(\mathbb{R}^{n}\right)$ is dense in $H^{m, p}\left(\mathbb{R}^{n}\right)$, it is easy to see that $U$ satisfies (1.1).

To prove that $U \equiv 0$ it is sufficient to prove that there is a $\rho>0$ so that $U \equiv 0$ in the strip $S_{\rho}=\left\{x \in \mathbb{R}^{n}: x_{1} \leq \rho\right\}$. Take $\rho>0$ so small that, if $V$ is as above and $C$ is as in (0.2),

$$
C\|V\|_{L^{\frac{n}{m}}\left(S_{\rho}\right)} \leq \frac{1}{2}
$$

If one uses Hölder's inequality, along with (1.1) and (1.2), and the fact that $|P(D) U| \leq|V U|$, then one has the following string of inequalities for every 
$\tau>0$

$$
\begin{aligned}
\left\|e^{-\tau x_{1}} U\right\|_{L^{p^{\prime}}\left(S_{\rho}\right)} & \leq C\left\|e^{-\tau x_{1}} P(D) U\right\|_{L^{p}\left(\mathbb{R}^{n}\right)} \\
& \leq C\left\|e^{-\tau x_{1}} V U\right\|_{L^{p}\left(S_{\rho}\right)}+C\left\|e^{-\tau x_{1}} P(D) U\right\|_{L^{p}\left(\mathbb{R}^{n} / S_{\rho}\right)} \\
& \leq \frac{1}{2}\left\|e^{-\tau x_{1}} U\right\|_{L^{p^{\prime}}\left(S_{\rho}\right)}+C\left\|e^{-\tau x_{1}} P(D) U\right\|_{L^{p}\left(\mathbb{R}^{n} / S_{\rho}\right)} .
\end{aligned}
$$

Hence,

$$
\left\|e^{-\tau x_{1}} U\right\|_{L^{p^{\prime}}\left(S_{\rho}\right)} \leq 2 C\left\|e^{-\tau x_{1}} P(D) U\right\|_{L^{p}\left(\mathbb{R}^{n} / S_{\rho}\right)},
$$

and consequently,

$$
\left\|e^{\tau\left(\rho-x_{1}\right)} U\right\|_{L^{p^{\prime}\left(S_{\rho}\right)}} \leq 2 C\|P(D) U\|_{L^{p}\left(\mathbb{R}^{n}\right)} .
$$

Since the above inequality holds for every $\tau>0$, this forces $U \equiv 0$ in $S_{\rho}$.

Proof of Theorem 1. Consider the operators

$$
\begin{aligned}
T_{j} f(x)=\int_{\mathbb{R}^{n}} \int_{\mathbb{R}^{n}} \frac{f(y) e^{i\langle x-y, \zeta\rangle}}{\left(\zeta_{1}-\lambda_{j}\left(\zeta^{\prime}\right)\right)+i\left(1-\mu_{j}\left(\zeta^{\prime}\right)\right)} \chi(\zeta) d \zeta d y, & \\
f \in \mathcal{S}\left(\mathbb{R}^{n}\right), \quad j=1, \ldots, & \frac{m}{2} .
\end{aligned}
$$

(0.5) can thus be rewritten as

$$
\left\|T_{j} f\right\|_{p^{\prime}} \leq C\|f\|_{p}, \quad f \in \mathcal{S}\left(\mathbb{R}^{n}\right), \quad j=1, \ldots, \frac{m}{2} .
$$

For simplicity, we will omit the subscript $j$ from now on. The change of variables $\zeta_{1} \rightarrow \zeta_{1}+\lambda\left(\zeta^{\prime}\right)$ allows to write

$$
T f(x)=\int_{\mathbb{R}^{n}} f(y)\left(\int_{\mathbb{R}^{n}} \frac{e^{i\left(\langle x-y, \zeta\rangle+\lambda\left(\zeta^{\prime}\right)\left(x_{1}-y_{1}\right)\right)}}{\zeta_{1}+i\left(1-\mu\left(\zeta^{\prime}\right)\right)} \chi\left(\zeta_{1}, \zeta^{\prime}\right) d \zeta\right) d y,
$$

and if we set $\zeta_{1}\left(1-\mu\left(\zeta^{\prime}\right)\right)^{-1}=t$, and we assume that $\chi\left(t\left(1-\mu\left(\zeta^{\prime}\right)\right), \zeta^{\prime}\right)=$ $\chi(t) \chi\left(\zeta^{\prime}\right)$,

$T f(x)=\int_{\mathbb{R}^{n}} f(y) \int_{\mathbb{R}^{n-1}} e^{i\left(\left\langle x^{\prime}-y^{\prime}, \zeta^{\prime}\right\rangle+\lambda\left(\zeta^{\prime}\right)\left(x_{1}-y_{1}\right)\right)} h\left(\left(x_{1}-y_{1}\right)\left(1-\mu\left(\zeta^{\prime}\right)\right) \chi\left(\zeta^{\prime}\right)\right) d \zeta^{\prime}$,

where

$$
h(s)=\int_{\mathbb{R}} \frac{e^{i t s}}{t+i} \chi(t) d t
$$


Define the "frozen operator"

$$
T_{x_{1}-y_{1}} g\left(x^{\prime}\right)=\int_{\mathbb{R}^{n-1}} \hat{g}\left(\zeta^{\prime}\right) h\left(\left(x_{1}-y_{1}\right)\left(1-\mu\left(\zeta^{\prime}\right)\right)\right) e^{i\left(\left\langle x^{\prime}, \zeta^{\prime}\right\rangle+\lambda\left(\zeta^{\prime}\right)\left(x_{1}-y_{1}\right)\right)} \chi\left(\zeta^{\prime}\right) d \zeta^{\prime} .
$$

If we prove that $T_{x_{1}-y_{1}}$ extends to a bounded operator from $L^{p}\left(\mathbb{R}^{n-1}\right)$ to $L^{p^{\prime}}\left(\mathbb{R}^{n-1}\right)$ with norm $O\left(\left|x_{1}-y_{1}\right|^{-\beta}\right)$,

$$
\beta \geq 1+\frac{1}{p^{\prime}}-\frac{1}{p}
$$

then we are done. In fact, $\operatorname{Tf}(x)=\int_{\mathbb{R}} T_{x_{1}-y_{1}} f\left(y_{1}, x^{\prime}\right) d y_{1}$, and hence

$$
\begin{aligned}
\left\|T f\left(x_{1}, .\right)\right\|_{L^{p^{\prime}}\left(\mathbb{R}^{n-1}\right)} & \leq \int_{\mathbb{R}}\left\|T_{x_{1}-y_{1}} f\left(y_{1}, .\right)\right\|_{L^{p^{\prime}\left(\mathbb{R}^{n-1}\right)}} d y_{1} \\
& \leq C \int_{\mathbb{R}}\left\|f\left(y_{1}, .\right)\right\|_{L^{p}\left(\mathbb{R}^{n-1}\right)}\left|x_{1}-y_{1}\right|^{-\beta} d y_{1} .
\end{aligned}
$$

By the Hardy-Littlewood-Sobolev inequality,

$$
x_{1} \rightarrow \int_{\mathbb{R}}\left\|f\left(y_{1}, .\right)\right\|_{L^{p}\left(\mathbb{R}^{n-1}\right)}\left|x_{1}-y_{1}\right|^{-\beta} d y_{1} \in L^{p^{\prime}}(\mathbb{R})
$$

when $\beta$ is as in (1.4), and

$$
\begin{aligned}
& \left\|\int_{\mathbb{R}}\right\| f\left(y_{1}, .\right)\left\|_{L^{p}\left(\mathbb{R}^{n-1}\right)}\left|x_{1}-y_{1}\right|^{-\beta} d y_{1}\right\|_{L^{p^{\prime}\left(\mathbb{R}, d x_{1}\right)}} \\
& \leq C\left(\int_{\mathbb{R}}\left\|f\left(y_{1}, .\right)\right\|_{L^{p}\left(\mathbb{R}^{n-1}\right)}^{p} d y_{1}\right)^{\frac{1}{p}}=C\|f\|_{p} .
\end{aligned}
$$

By (1.5) and the above, $\|T f\|_{p^{\prime}} \leq C\|f\|_{p}$.

We are thus left to estimate $\left\|T_{x_{1}-y_{1}} g\right\|_{L^{p^{\prime}\left(\mathbb{R}^{n-1}\right)}}$. If we let $\Sigma=\left\{\zeta^{\prime} \in\right.$ $\left.\mathbb{R}^{n-1}: \mu\left(\zeta^{\prime}\right)=1\right\}$, and use polar coordinates associated to $\Sigma$,

$$
\begin{aligned}
& T_{x_{1}-y_{1}} g\left(x^{\prime}\right) \\
& =\int_{0}^{+\infty} r^{n-2} \int_{\Sigma} g(r \omega) h\left(\left(x_{1}-y_{1}\right)(1-r)\right) \chi(r \omega) e^{i r\left(\left\langle x^{\prime}, \omega\right\rangle+\left(x_{1}-y_{1}\right) \lambda(\omega)\right)} d \omega d r .
\end{aligned}
$$

Without loss of generality, $r^{n-2} \chi(r \omega)=\chi(r) \chi(\omega)$. Then,

$$
T_{x_{1}-y_{1}} g\left(x^{\prime}\right)=\int_{0}^{+\infty} \chi(r) h\left(\left(x_{1}-y_{1}\right)(1-r)\right) I_{x_{1}-y_{1}, r} g\left(x^{\prime}\right) d r
$$

where we have set

$$
I_{x_{1}-y_{1}, r} g\left(x^{\prime}\right)=\int_{\Sigma} \hat{g}(r \omega) e^{i r\left(\left\langle x^{\prime}, \omega\right\rangle+\left(x_{1}-y_{1}\right) \lambda(\omega)\right)} \chi(\omega) d \sigma(\omega) .
$$


By Minkowsky's inequality,

$$
\left\|T_{x_{1}-y_{1}} g\right\|_{L^{p^{\prime}\left(\mathbb{R}^{n-1}\right)}} \leq \int_{0}^{+\infty} \chi(r)\left|h\left(\left(x_{1}-y_{1}\right)(1-r)\right)\right|\left\|I_{x_{1}-y_{1}, r} g\right\|_{L^{p^{\prime}\left(\mathbb{R}^{n-1}\right)}} d r .
$$

If $I_{x_{1}-y_{1}, r}$ maps $L^{p}\left(\mathbb{R}^{n-1}\right)$ boundedly into $L^{p^{\prime}}\left(\mathbb{R}^{n-1}\right)$, then

$$
\left\|T_{x_{1}-y_{1}} g\right\|_{L^{p^{\prime}\left(\mathbb{R}^{n-1}\right)}} \leq C\|g\|_{L^{p}\left(\mathbb{R}^{n-1}\right)} \int_{0}^{+\infty} \chi(r)\left|h\left(\left(x_{1}-y_{1}\right)(1-r)\right)\right| d r
$$

and, by the definition of $h,\left\|T_{x_{1}-y_{1}} g\right\|_{L^{p^{\prime}\left(\mathbb{R}^{n-1}\right)}} \leq C\left|x_{1}-y_{1}\right|^{-1}\|g\|_{L^{p}\left(\mathbb{R}^{n-1}\right)}$, which is even better than what we need.

Let $\beta \in C_{0}^{\infty}(\mathbb{R})$ be an even and nonnegative function whose support is concentrated in a neighborhood of the origin and such that $\int_{\mathbb{R}} \beta(t) d t=1$. Let

$$
I_{x_{1}-y_{1}, r}^{\epsilon} g\left(x^{\prime}\right)=\frac{1}{\epsilon} \int_{\mathbb{R}^{n-1}} \hat{g}(\eta) \beta\left(\frac{|r-\mu(\eta)|}{r \epsilon}\right) e^{i\left(\left\langle x^{\prime}, \eta\right\rangle+\left(x_{1}-y_{1}\right) \lambda(\eta)\right)} \chi(\eta) d \eta .
$$

If we prove that

(i) $\left\|I_{x_{1}-y_{1}, r}^{\epsilon} g\right\|_{L^{p^{\prime}\left(\mathbb{R}^{n-1}\right)}} \leq C\|g\|_{L^{p}\left(\mathbb{R}^{n-1}\right)}, g \in \mathcal{S}\left(\mathbb{R}^{n-1}\right)$, with $C$ independent of $\epsilon$,

(ii) $\quad I_{x_{1}-y_{1}, r}^{\epsilon} g \rightarrow I_{x_{1}-y_{1}, r} g$ in distribution sense as $\epsilon \rightarrow 0$,

then $\left\|I_{x_{1}-y_{1}, r} g\right\|_{L^{p^{\prime}\left(\mathbb{R}^{n-1}\right)}} \leq C\|g\|_{L^{p}\left(\mathbb{R}^{n-1}\right)}$. Recalling that the spheres of $L^{p^{\prime}}\left(\mathbb{R}^{n-1}\right)$ are weakly sequentially compact, from (i) follows in fact that, for every $g \in \mathcal{S}\left(\mathbb{R}^{n}\right)$, there exists a sequence $\left\{I_{x_{1}-y_{1}, r}^{\epsilon_{j}}(g)\right\}_{j \in \mathbb{N}}$ which converges in the weak topology of $L^{p^{\prime}}\left(\mathbb{R}^{n-1}\right)$. By (ii), $I_{x_{1}-y_{1}, r}^{\epsilon_{j}} g \rightarrow I_{x_{1}-y_{1}, r} g$, and by (i), $\left\|I_{x_{1}-y_{1}, r} g\right\|_{L^{p^{\prime}}} \leq C\|g\|_{L^{p}}$.

To prove (i) it is convenient to write $I_{x_{1}-y_{1}, r}^{\epsilon}$ as the composition of two operators, and study them separately.

Let

$$
\begin{aligned}
G_{r}^{\epsilon} g\left(x^{\prime}\right) & =\frac{1}{\sqrt{\epsilon}} \int_{\mathbb{R}^{n-1}} \hat{g}(\eta) \beta^{\frac{1}{2}}\left(\frac{|r-\mu(\eta)|}{r \epsilon}\right) e^{i\left\langle x^{\prime}, \eta\right\rangle} \chi(\eta) d \eta, \\
Q_{x_{1}-y_{1}, r}^{\epsilon} g\left(x^{\prime}\right) & =\frac{1}{\sqrt{\epsilon}} \int_{\mathbb{R}^{n-1}} \hat{g}(\eta) \beta^{\frac{1}{2}}\left(\frac{|r-\mu(\eta)|}{r \epsilon}\right) e^{i\left(\left\langle x^{\prime}, \eta\right\rangle+\left(x_{1}-y_{1}\right) \lambda(\eta)\right)} \chi(\eta) d \eta .
\end{aligned}
$$

Since $I_{x_{1}-y_{1}, r}^{\epsilon}=G_{r}^{\epsilon} \circ Q_{x_{1}-y_{1}, r}^{\epsilon}$, for a suitable choice of the cutoff function $\chi$, and $\left(G_{r}^{\epsilon}\right)^{*}=G_{r}^{\epsilon}$, to prove that $I_{x_{1}-y_{1}, r}^{\epsilon}: L^{p}\left(\mathbb{R}^{n-1}\right) \rightarrow L^{p^{\prime}}\left(\mathbb{R}^{n-1}\right)$ is a bounded operator, it is enough to prove that $G_{r}^{\epsilon}$ and $Q_{x_{1}-y_{1}, r}^{\epsilon} \operatorname{map} L^{p}\left(\mathbb{R}^{n-1}\right)$ boundedly into $L^{2}\left(\mathbb{R}^{n-1}\right)$. 
Consider $Q_{x_{1}-y_{1}, r}^{\epsilon}$ first. Since the function $\zeta^{\prime} \rightarrow e^{i \lambda\left(\zeta^{\prime}\right)\left(x_{1}-y_{1}\right)}$ is bounded, it is a $L^{2}$ Fourier multiplier, (see e.g. $[\mathbf{H}]$ ). Hence,

$$
\left\|Q_{x_{1}-y_{1}, r}^{\epsilon} g\right\|_{L^{2}} \leq \frac{1}{\sqrt{\epsilon}}\left\|\int_{\mathbb{R}^{n-1}} \hat{g}(\eta) \beta^{\frac{1}{2}}\left(\frac{|r-\mu(\eta)|}{r \epsilon}\right) e^{i\left\langle x^{\prime}, \eta\right\rangle} \chi(\eta) d \eta\right\|_{L^{2}},
$$

and by the Plancherel theorem,

$$
\left\|Q_{x_{1}-y_{1}, r}^{\epsilon} g\right\|_{L^{2}} \leq\left(\frac{1}{\epsilon} \int_{\mathbb{R}^{n-1}}|\hat{g}(\eta)|^{2} \beta\left(\frac{|r-\mu(\eta)|}{r \epsilon}\right) \chi^{2}(\eta) d \eta\right)^{\frac{1}{2}} .
$$

Let $Q_{\epsilon}^{\prime} g$ denote the right-hand side of (1.8). In polar coordinates associated to $\Sigma$,

$$
Q_{\epsilon}^{\prime} g\left(x^{\prime}\right)=\left(\frac{1}{\epsilon} \int_{0}^{+\infty} \beta\left(\frac{|r-s|}{r \epsilon}\right) \int_{\Sigma}|\hat{g}(s \omega)|^{2} \chi^{2}(s \omega) d \omega d s\right)^{\frac{1}{2}} .
$$

Without loss of generality, $\chi^{2}(s \omega)=\chi(\omega) \chi(s)$. Since $\hat{g}(s \omega)=s^{-n-1} \hat{g_{s}}(\omega)$, where $g_{s}(\omega)=g\left(\frac{\omega}{s}\right)$, if we let $t=\frac{r-s}{r}$,

$$
Q_{\epsilon}^{\prime} g\left(x^{\prime}\right)=\left(\frac{1}{\epsilon r^{2(n-1)}} \int_{\mathbb{R}} \beta\left(\frac{|t|}{\epsilon}\right) \frac{\chi(t)}{(1-t)^{2(n-1)}} \int_{\Sigma}\left|\hat{g}_{r(1-t)}(\omega)\right|^{2} \chi(\omega) d \omega d t\right)^{\frac{1}{2}} .
$$

By the Assumption (2), $\Sigma$ has nonvanishing Gaussian curvature on the support of $\chi$. We can apply Theorem $\mathbf{A}$ in the Appendix to obtain the following string of inequalities:

$$
\begin{aligned}
& \left\|Q_{\epsilon}^{\prime} g\right\|_{L^{2}\left(\mathbb{R}^{n-1}\right)} \\
& \leq C\left(\frac{1}{\epsilon r^{2(n-1)}} \int_{\mathbb{R}} \beta\left(\frac{|t|}{\epsilon}\right)\left\|g_{r(1-t)}\right\|_{L^{p}\left(\mathbb{R}^{n-1}\right)}^{2} \frac{\chi(t)}{(1-t)^{2(n-1)}} d t\right)^{\frac{1}{2}} \\
& =C r^{\frac{n-1}{p^{\prime}}}\|g\|_{p}\left(\frac{1}{\epsilon} \int_{\mathbb{R}} \chi(t)(1-t)^{\frac{2(n-1)}{p^{\prime}}} \beta\left(\frac{|t|}{\epsilon}\right) d t\right)^{\frac{1}{2}} .
\end{aligned}
$$

The family of functions $\left\{\frac{1}{\epsilon} \beta\left(\frac{|t|}{\epsilon}\right)\right\}_{\epsilon>0}$ converges to the Dirac distribution $\delta_{0}$ in distribution sense. Hence,

$$
\left\|Q_{\epsilon}^{\prime} g\right\|_{L^{2}\left(\mathbb{R}^{n-1}\right)} \leq C r^{\frac{n-1}{p^{\prime}}}\|g\|_{L^{p}\left(\mathbb{R}^{n-1}\right)} .
$$

The same technique applies to estimate $\left\|G_{r}^{\epsilon} g\right\|_{L^{2}\left(\mathbb{R}^{n-1}\right)}$. (i) is thus proved.

We now prove (ii). For $\phi, g \in \mathcal{S}\left(\mathbb{R}^{n}\right)$,

$$
\left\langle I_{x_{1}-y_{1}, r}^{\epsilon} g, \phi\right\rangle=\frac{1}{\epsilon} \int_{\mathbb{R}^{n-1}} \hat{g}(\eta) \hat{\phi}(\eta) \beta\left(\frac{|r-\mu(\eta)|}{r \epsilon}\right) e^{i\left(x_{1}-y_{1}\right) \lambda(\eta)} \chi(\eta) d \eta .
$$


If we set $\eta=r \zeta^{\prime}$,

$$
\begin{aligned}
& \left\langle I_{x_{1}-y_{1}, r}^{\epsilon} g, \phi\right\rangle \\
& =\frac{r^{n-1}}{\epsilon} \int_{\mathbb{R}^{n-1}} \hat{g}\left(r \zeta^{\prime}\right) \hat{\phi}\left(r \zeta^{\prime}\right) \beta\left(\frac{\left|1-\mu\left(\zeta^{\prime}\right)\right|}{\epsilon}\right) e^{i r\left(x_{1}-y_{1}\right) \lambda\left(\zeta^{\prime}\right)} \chi\left(r \zeta^{\prime}\right) d \zeta^{\prime},
\end{aligned}
$$

and we use polar coordinates associated to $\Sigma$,

$$
\frac{1}{\epsilon} \int_{\mathbb{R}} \chi(t) \beta\left(\frac{|1-t|}{\epsilon}\right) \int_{\Sigma} \hat{g}(r t \omega) \hat{\phi}(r t \omega) e^{i r t\left(x_{1}-y_{1}\right) \lambda(\omega)} \chi(r t \omega) d \omega d t .
$$

The family of functions $\left\{\frac{1}{\epsilon} \beta\left(\frac{|1-t|}{\epsilon}\right)\right\}_{\epsilon>0}$ converges to the Dirac distribution $\delta_{1}$ in distribution sense. Hence,

$$
\begin{aligned}
\lim _{\epsilon \rightarrow 0^{+}} & =\left\langle I_{x_{1}-y_{1}, r}^{\epsilon} g, \phi\right\rangle=\int_{\Sigma} \hat{g}(r \omega) \hat{\phi}(r \omega) e^{i r\left(x_{1}-y_{1}\right) \lambda(\omega)} \chi(\omega) d \sigma \omega \\
& =\int_{\mathbb{R}^{n-1}} I_{x_{1}-y_{1}, r} g\left(x^{\prime}\right) \phi\left(x^{\prime}\right) d x^{\prime}=\left\langle I_{x_{1}-y_{1}, r} g, \phi\right\rangle .
\end{aligned}
$$

This concludes the proof of the theorem.

Remark. If $\Sigma$ does not have everywhere nonvanishing Gaussian curvature, but has has at least $k$ nonvanishing principal curvatures, the thesis of Theorem 1 holds for $p \leq \frac{2(k+2)}{k+4}$. The proof of the above result is the same as the proof of Theorem 1, where one uses Theorem B in the appendix in place of Theorem A.

\section{Appendix.}

Let $S$ denote a smooth hypersurface of $\mathbb{R}^{n}, n \geq 3$, equiped with a smooth compactly supported measure $d \mu$. Let $J: S \rightarrow S^{N}$ be the usual Gauss map taking each point on $S$ to the outward unit normal at that point. We say that $S$ has everywhere nonvanishing Gaussian curvature if the differential of the Gauss map $d J$ is always nonsingular. We say that $S$ has at least $k$ nonvanishing principal curvatures if the rank of $d J$ is always $\geq k$

Theorem A. If $S$ has everywhere nonvanishing Gaussian curvature, the following inequality holds for $p \leq \frac{2(n+1)}{n+3}$ :

$$
\left(\int_{S}|\hat{f}(\xi)|^{2} d \mu(s)\right)^{\frac{1}{2}} \leq C\|f\|_{L^{p}\left(\mathbb{R}^{n}\right)}, \quad \quad f \in C_{0}^{\infty}\left(\mathbb{R}^{n}\right)
$$

Proof. See e.g. [S], p. 60. 
From a theorem of Littman $[\mathbf{L}]$, and one of Greenleaf $[\mathbf{G}]$, one can prove the following generalization of Theorem A:

Theorem B. If $S$ has at least $k$ nonvanishing principal curvature everywhere, the following inequality holds for $p \leq \frac{2(k+2)}{k+4}$ :

$$
\left(\int_{S}|\hat{f}(\xi)|^{2} d \mu(\xi)\right)^{\frac{1}{2}} \leq C\|f\|_{L^{p}\left(\mathbb{R}^{n}\right)}, \quad f \in C_{0}^{\infty}\left(\mathbb{R}^{n}\right) .
$$

\section{References}

[DC] L. De Carli, Unique continuation for higher order elliptic operators, Ph.D. thesis, UCLA, 1993.

[G] A. Greenleaf, Principal curvatures in harmonic analysis, Ind. Univ. Math. J., 30 (1981), 519-537.

[H] L. Hörmander, The analysis of linear partial differential operators, Vol. 4, Springer Verlag, 1983.

[H1] Uniqueness theorems for second order elliptic differential operators, Comm. P.D.E., 8 (1983), 21-64.

[H2] - On the uniqueness of the Cauchy problem, Math. Scand., 6 (1958), 213-225.

[J] D. Jerison, Carleman inequalities for the Dirac and Laplace operator and unique continuation, Adv. Math., 63 (1986), 118-134.

[JK] D. Jerison and C. Kenig, Unique continuation and absence of positive eigenvalues for Schrödinger operators, Ann. of Math., 121 (1985), 463-494.

[KRS] C.E. Kenig, A. Ruiz and C.D. Sogge, Uniform Sobolev estimates and unique continuation for second order constant coefficient differential operators, Duke Math. J., $\mathbf{5 5}(\mathbf{2})$ (1987), 329-347.

[L] W. Littman, Fourier transforms of surface-carried measures and differentiability of surface averages, Bull. Am. Math. Soc., 69 (1963), 766-770.

[P] A. Plis, A smooth linear elliptic differential equation without any solution in a sphere, Comm. Pure Appl. Math., 14 (1961), 599-617.

[S] C.D.Sogge, Fourier integrals in classical analysis, Cambridge University Press, 1993.

[S1] - Oscillatory integrals and unique continuation for second order elliptic differential equations, J. Am. Math. Soc., 2 (1989), 491-516.

Received September 28, 1993 and revised November 20, 1995.

UNIVERSITA ${ }^{\prime}$ DEGLI STUDI DI NAPOLI

80126 NAPOLI, ITALY

E-mail address: decarli@matna2.dma.unina.it 